\title{
Strong Converse Inequalities and Quantitative Voronovskaya-Type Theorems for Trigonometric Fejér Sums
}

\author{
JORGE BUSTAMANTE* AND LÁZARO FLORES-DE-JESÚS
}

ABSTRACT. Let $\sigma_{n}$ denotes the classical Fejér operator for trigonometric expansions. For a fixed even integer $r$, we characterize the rate of convergence of the iterative operators $\left(I-\sigma_{n}\right)^{r}(f)$ in terms of the modulus of continuity of order $r$ (with specific constants) in all $\mathbb{L}^{p}$ spaces $1 \leq p \leq \infty$. In particular, the constants depend not on $p$. Moreover, we present a quantitative version of the Voronovskaya-type theorems for the operators $\left(I-\sigma_{n}\right)^{r}(f)$.

Keywords: Fejér operators, iterative combinations, rate of convergence, direct and converse results, quantitative Voronovskaya-type theorems.

2010 Mathematics Subject Classification: 42A10, 42A20, 41A25, 41A27.

\section{INTRODUCTION}

Let $C_{2 \pi}$ denote the Banach space of all $2 \pi$-periodic, continuous functions $f$ defined on the real line $\mathbb{R}$ with the sup norm

$$
\|f\|_{\infty}=\max _{x \in[-\pi, \pi]}|f(x)| .
$$

For $1 \leq p<\infty$, the Banach space $\mathbb{L}^{p}$ consists of all $2 \pi$-periodic, $p$-th power Lebesgue integrable functions $f$ on $\mathbb{R}$ with the norm

$$
\|f\|_{p}=\left(\frac{1}{2 \pi} \int_{-\pi}^{\pi}|f(x)|^{p} d x\right)^{1 / p} .
$$

In order to simplify, we write $X^{p}=\mathbb{L}^{p}$ for $1 \leq p<\infty$ and $X^{\infty}=C_{2 \pi}$. As usual, for $r \in \mathbb{N}$, by $W_{p}^{r}$ we mean the family of all functions $f \in X^{p}$ such that $f, \ldots, D^{r-1}(f)$ are absolutely continuous and $D^{r}(f) \in X^{p}$. Here $D(f)=D^{1}(f)=f^{\prime}$ and $D^{r+1}(f)=D\left(D^{r}(f)\right)$.

For $f \in X^{1}$, the conjugate function is defined by

$$
\tilde{f}(x)=-\frac{1}{2 \pi} \int_{0}^{\pi} \frac{f(x+t)-f(x-t)}{\tan (t / 2)} d t=-\lim _{\varepsilon \rightarrow 0} \frac{1}{2 \pi} \int_{\varepsilon}^{\pi} \frac{f(x+t)-f(x-t)}{\tan (t / 2)} d t,
$$

whenever the limit exists. It is know that if $f \in X^{p}$ with $1<p<\infty$, then $\tilde{f} \in X^{p}$, and that is not the case for $p=1$ and $p=\infty$.

For $r \in \mathbb{N}$, function $f \in X^{p}$, and $h>0$, the usual modulus of smoothness of order $r$ of $f$ is defined by

$$
\omega_{r}(f, t)_{p}=\sup _{|h| \leq t}\left\|\left(I-T_{h}\right)^{r}(f)\right\|_{p}
$$

Received: 02.12.2019; Accepted: 05.03.2020; Published Online: 06.03.2020

*Corresponding author: Jorge Bustamante; jbusta@fcfm.buap.mx

DOI: $10.33205 / \mathrm{cma} .653843$ 
where $T_{h}(f, x)=f(x+h)$ is the translation operator. We also use the notations $\Delta_{h}^{r} f(x)=$ $\left(I-T_{h}\right)^{r}(f)$.

Let $\mathbb{T}_{n}$ denote the family of all real trigonometric polynomials of degree not greater than $n$. For $1 \leq p \leq \infty$ and $f \in X^{p}$, the best approximation of $f$ by elements of $\mathbb{T}_{n}$ is defined by

$$
E_{n, p}(f)=\inf _{T \in \mathbb{T}_{n}}\|f-T\|_{p}
$$

Recall that for $f \in \mathbb{L}^{1}$ and $k \in \mathbb{N}_{0}$, the Fourier coefficients are defined by

$$
a_{k}(f)=\frac{1}{\pi} \int_{-\pi}^{\pi} f(t) \cos (k t) d t \quad \text { and } \quad b_{k}(f)=\frac{1}{\pi} \int_{-\pi}^{\pi} f(t) \sin (k t) d t,
$$

and the (formal) Fourier series is given by

$$
f(x) \sim \frac{a_{0}(f)}{2}+\sum_{k=1}^{\infty}\left(a_{k}(f) \cos (k x)+b_{k}(f) \sin (k x)\right)=\sum_{k=0}^{\infty} A_{k}(f, x) .
$$

For $n \in \mathbb{N}$ and $f \in X^{1}$, the Fejér sum of order $n$ is defined by

$$
\sigma_{n}(f, x)=\sum_{k=0}^{n}\left(1-\frac{k}{n+1}\right) A_{k}(f, x) .
$$

We also consider the conjugate operators

$$
\widetilde{\sigma}_{n}(f, x)=\sum_{k=1}^{n}\left(1-\frac{k}{n+1}\right) B_{k}(f, x),
$$

where $B_{k}(f, x)=-b_{k}(f) \cos (k x)+a_{k}(f) \sin (k x)$.

In what follows the following notations are used: $\sigma_{n}^{0}=I$ (identity operator), $\sigma_{n}^{1}(f)=\sigma_{n}(f)$ and $\sigma_{n}^{r+1}=\sigma_{n}\left(\sigma_{n}^{r}(f)\right)$.

In [3], it was proved that if $1 \leq p \leq \infty, n>1$, and $f \in X^{p}$, then

$$
\frac{1}{2}\left\|f-\sigma_{n}(f)\right\|_{p} \leq \widetilde{K}\left(f, \frac{3}{n}\right)_{p} \leq 4\left\|f-\sigma_{n}(f)\right\|_{p},
$$

where

$$
\widetilde{K}(f, t)_{p}=\inf \left\{\|f-g\|_{p}+t\|D(\widetilde{g})\|_{p}: g \in X^{p}, \widetilde{g} \in A C, D(\widetilde{g}) \in X^{p}\right\},
$$

here $D(h)=h^{\prime}$. Moreover, for $1<p<\infty$, it was given a constant $C_{p}$ such that, for each $f \in X^{p}$,

$$
\frac{1}{2 C_{p}}\left\|f-\sigma_{n}(f)\right\|_{p} \leq \omega_{1}\left(f, \frac{1}{n}\right)_{p} \leq 8 C_{p}\left\|f-\sigma_{n}(f)\right\|_{p} .
$$

On the other hand, in recent times there have been some interests in studying quantitative Voronovskaya-type theorems, but almost all the papers concern with positive linear operators in spaces of non-periodic functions. For instance; see [1], [2], [11], [12] and the references therein. The methods used in those papers are not useful in dealing with periodic functions for two reasons (at least). First, they use different kinds of Taylor's formula and second, in the non-periodical case do not appear conjugate functions.

It is known that the Voronovskaya-type theorems are related with the saturation class of some families of operators. We have noticed that in the case of trigonometric polynomial approximation process the Voronovskaya-type theorems depend on particular properties of the operators. In this paper we consider the Fejér operators (other approximation methods will be studied in forthcoming papers). 
In this paper, two different kind of results are presented. First, we extend equation (1.2) by proving that, for an even integer $r, f \in X^{p}$ and $n \in \mathbb{N}$,

$$
C_{1} \omega_{r}\left(f, \frac{\pi}{n}\right)_{p} \leq\left\|\left(I-\sigma_{n}\right)^{r}(f)\right\|_{p} \leq C_{2} \omega_{r}\left(f, \frac{\pi}{n}\right)_{p} .
$$

Second, we verify a quantitative Voronovskaya-type relation in the following form: $1 \leq p \leq \infty$, $r$ is an even integer, $f \in W_{p}^{r}$, and $n \geq r$, then

$$
\left\|(n+1)^{r}\left(I-\sigma_{n}\right)^{r}(f)-(-1)^{r / 2} D^{r}(f)\right\|_{p} \leq C_{3} E_{n, p}\left(D^{r}(f)\right) .
$$

Here $C_{1}, C_{2}$ and $C_{3}$ are positive constants which will be given in an explicit form. These tasks will be accomplished in Sections 2 and 4, where other results are included. In order to present the estimate in Section 4 with specific constants, we need some results related with simultaneous approximation that will be proved in Section 3.

\section{STRONG INEQUALITIES FOR COMBINATIONS OF FEJÉR OPERATORS}

First, we recall some known results that will be needed later.

Proposition 2.1. (i) If $r \in \mathbb{N}, t>0$ and $f \in X^{p}$, then $\omega_{r}(f, t)_{p} \leq 2^{r}\|f\|_{p}$.

(ii) ([15, page 103]) If $r \in \mathbb{N}$ and $f \in W_{p}^{r}$, then

$$
\omega_{r}(f, t) \leq t^{r}\left\|D^{r}(f)\right\|_{p} .
$$

(iii) ([15, page 103]) If $r, q \in \mathbb{N}$ and $f \in X^{p}$, then

$$
\omega_{r}(f, q t) \leq q^{r} \omega_{r}(f, q t) .
$$

For a proof of (2.5), see [15, page 215]. The first inequality in (2.6) is a consequence of (2.5) (take $h=\pi / n$ ) and Proposition 2.1, but for a direct proof see [15, page 208].

Theorem 2.1. If $1 \leq p \leq \infty, r, n \in \mathbb{N}$ and $T_{n} \in \mathbb{T}_{n}$, then

$$
\left\|T_{n}^{(r)}\right\|_{p} \leq\left(\frac{n}{2 \sin (n h / 2)}\right)^{r}\left\|\Delta_{h}^{r} T_{n}\right\|_{p},
$$

for any $h \in(0,2 \pi / n)$. Moreover

$$
\left\|D^{r}\left(T_{n}\right)\right\|_{p} \leq n^{r}\|T\|_{p} \quad \text { and } \quad\left\|D^{r}\left(\widetilde{T}_{n}\right)\right\|_{p} \leq n^{r}\left\|T_{n}\right\|_{p} .
$$

The first inequality in (2.6) is easily derived from (2.5) by taking $h=\pi / n$ and using the assertion (i) in Proposition 2.1. Unfortunately, the second inequality is not included in some books, but a proof can be seen in [14, page 135] (notice that it is sufficient to verify the assertion for $r=1$ ).

Proposition 2.2. (see [5, Cor. 1.2.4]) If $1 \leq p \leq \infty, n \in \mathbb{N}$, and $f \in X^{p}$, then

$$
\left\|\sigma_{n}(f)\right\|_{p} \leq\|f\|_{p}
$$

Let us show some algebraic relations related with iterates of Fejér operators.

Proposition 2.3. For each $n, r \in \mathbb{N}$ and $f \in \mathbb{L}^{1}$ one has

$$
\sigma_{n}^{r}(f, x)=\sum_{k=0}^{n}\left(1-\frac{k}{n+1}\right)^{r} A_{k}(f, x)
$$

and

$$
\left(I-\sigma_{n}\right)^{r}(f)=f-\sum_{k=0}^{n}\left(1-\frac{k^{r}}{(n+1)^{r}}\right) A_{k}(f) .
$$


Proof. By the orthogonality relations we know that, for $0 \leq k \leq n, A_{k}\left(\sigma_{n}(f)\right)=(1-k /(n+$ 1)) $A_{k}(f)$. For instance,

$$
\begin{aligned}
a_{k}\left(\sigma_{n}(f)\right) & =\frac{1}{\pi} \int_{-\pi}^{\pi} \sigma_{n}(f, t) \cos (k t) d t=\sum_{k=0}^{n}\left(1-\frac{k}{n+1}\right) \frac{1}{\pi} \int_{-\pi}^{\pi} A_{k}(f, t) \cos (k t) d t \\
& =\left(1-\frac{k}{n+1}\right) \frac{1}{\pi} \int_{-\pi}^{\pi} a_{k}(f) \cos ^{2}(k t) d t=\left(1-\frac{k}{n+1}\right) a_{k}(f) .
\end{aligned}
$$

With similar arguments, we can verified that $b_{k}\left(\sigma_{n}(f)\right)=(1-k /(n+1)) b_{k}(f)$.

Hence

$$
\sigma_{n}^{2}(f)=\sum_{k=0}^{n}\left(1-\frac{k}{n+1}\right) A_{k}\left(\sigma_{n}(f)\right)=\sum_{k=0}^{n}\left(1-\frac{k}{n+1}\right)^{2} A_{k}(f) .
$$

For other values of $r$, the proof follows by induction.

On the other hand,

$$
\begin{aligned}
\left(I-\sigma_{n}\right)^{r}(f) & =\sum_{j=0}^{r}(-1)^{j}\left(\begin{array}{l}
r \\
j
\end{array}\right) \sigma_{n}^{j}(f)=f+\sum_{j=1}^{r}(-1)^{j}\left(\begin{array}{l}
r \\
j
\end{array}\right) \sigma_{n}^{j}(f) \\
& =f+\sum_{j=1}^{r}(-1)^{j}\left(\begin{array}{l}
r \\
j
\end{array}\right) \sum_{k=0}^{n}\left(1-\frac{k}{n+1}\right)^{j} A_{k}(f) \\
& =f+\sum_{k=0}^{n}\left(\sum_{j=1}^{r}\left(\begin{array}{l}
r \\
j
\end{array}\right)\left(\frac{k}{n+1}-1\right)^{j}\right) A_{k}(f) \\
& =f-\sum_{k=0}^{n} A_{k}(f)+\sum_{k=0}^{n}\left(\sum_{j=0}^{r}\left(\begin{array}{l}
r \\
j
\end{array}\right)\left(\frac{k}{n+1}-1\right)^{j}\right) A_{k}(f) \\
& \left.=f-\sum_{k=0}^{n} A_{k}(f)+\sum_{k=0}^{n}\left(\frac{k}{n+1}-1+1\right)^{r}\right) A_{k}(f) \\
& =f-\sum_{k=0}^{n}\left(1-\frac{k^{r}}{(n+1)^{r}}\right) A_{k}(f) .
\end{aligned}
$$

Proposition 2.4. For each $n, r \in \mathbb{N}$ and $T \in \mathbb{T}_{n}$ one has

$$
\left(I-\sigma_{n}\right)^{r}(T)=\left\{\begin{array}{cc}
\frac{(-1)^{r / 2}}{(n+1)^{r}} D^{r}(T), & r \text { even }, \\
\frac{(-1)^{(r-1) / 2}}{(n+1)^{r}} D^{r}(\widetilde{T}), & r \text { odd } .
\end{array}\right.
$$

Proof. It is easy to see that, for each polynomial $T \in \mathbb{T}_{n}$

$$
T-\sigma_{n}(T)=\frac{D(\widetilde{T})}{n+1} \quad \text { and } \quad\left(I-\sigma_{n}\right)^{2}(T)=-\frac{1}{(n+1)^{2}} D^{2}(T) .
$$

Since $\left(I-\sigma_{n}\right)^{r}$ is a linear operator, we can consider only the case $T(x)=a \cos (k x)+b \sin (k x)$, where $a, b \in \mathbb{R}$ and $1 \leq k \leq n$. In such a case from Proposition 2.3, we know that $\left(I-\sigma_{n}\right)^{r}(T)=$ $k^{r} T /(n+1)^{r}$.

If $r$ is even, by induction one has

$$
\left(I-\sigma_{n}\right)^{r+2}(T)=\frac{(-1)^{r / 2}}{(n+1)^{r}}\left(I-\sigma_{n}\right)^{2}\left(D^{r}(T)\right)=\frac{(-1)^{(r+2) / 2}}{(n+1)^{r}} D^{r+2}(T) .
$$


If $r>1$ is odd, taking into account (2.7) one has

$$
\begin{gathered}
\left(I-\sigma_{n}\right)^{r}(T)=\left(I-\sigma_{n}\right)^{r-1}\left(\left(I-\sigma_{n}\right)(T)\right)=\frac{1}{n+1}\left(I-\sigma_{n}\right)^{r-1}(D(\widetilde{T})) \\
=\frac{(-1)^{(r-1) / 2}}{(n+1)^{r}} D^{r-1}(D(\widetilde{T}))=\frac{(-1)^{(r-1) / 2}}{(n+1)^{r}} D^{r}(\widetilde{T}) .
\end{gathered}
$$

Theorem 2.2. If $1 \leq p \leq \infty, f \in X^{p}$ and $n, r \in \mathbb{N}$, with $r$ even, then

$$
\begin{aligned}
\frac{1}{2^{r}+\pi^{r}\left(2^{r}+1\right)} \omega_{r}( & \left.f, \frac{\pi}{n+1}\right)_{p} \leq\left\|\left(I-\sigma_{n}\right)^{r}(f)\right\|_{p} \\
& \leq(1+8 r(6+\ln r)) \omega_{r}\left(f, \frac{\pi}{n+1}\right)_{p} .
\end{aligned}
$$

Proof. (a) First inequality. If $f \in X^{p}$, fix $T \in \mathbb{T}_{n}$ such that $\|f-T\|_{p}=E_{n, p}(f)$.

Since $\left(I-\sigma_{n}\right)^{r}(f)-f \in \mathbb{T}_{n}$, one has

$$
E_{n, p}(f)=\|f-T\|_{p} \leq\left\|f-\left(\left(I-\sigma_{n}\right)(f)-f\right)\right\|_{p}=\left\|\left(I-\sigma_{n}\right)(f)\right\|_{p} .
$$

Thus, it follows from Propositions 2.1 and 2.4 that

$$
\begin{aligned}
\omega_{r}\left(f, \frac{\pi}{n+1}\right)_{p} \leq & 2^{r}\|f-T\|_{p}+\omega_{r}\left(T, \frac{\pi}{n+1}\right)_{p} \leq 2^{r}\|f-T\|_{p}+\frac{\pi^{r}}{(n+1)^{r}}\left\|D^{r}(T)\right\|_{p} \\
& =2^{r}\|f-T\|_{p}+\pi^{r}\left\|\left(I-\sigma_{n}\right)^{r}(T)\right\|_{p} \\
& \leq 2^{r}\|f-T\|_{p}+\pi^{r}\left(2^{r}\|f-T\|_{p}+\left\|\left(I-\sigma_{n}\right)^{r}(f)\right\|_{p}\right) \\
& \leq\left(2^{r}+\pi\left(2^{r}+1\right)\right)\left\|\left(I-\sigma_{n}\right)^{r}(f)\right\|_{p} .
\end{aligned}
$$

(b) Second inequality. Let $T$ be given as in part (a). Using Propositions 2.1, 2.4 and 2.3 and equation (2.5) (with $h=\pi / n$ ), one has

$$
\begin{aligned}
& \left\|\left(I-\sigma_{n}\right)^{r}(f)\right\|_{p} \leq\left\|\left(I-\sigma_{n}\right)^{r}(f-T)\right\|_{p}+\left\|\left(I-\sigma_{n}\right)^{r}(T)\right\|_{p} \\
& \leq 2^{r} E_{n, p}(f)+\frac{1}{(n+1)^{r}}\left\|D^{r}(T)\right\|_{p} \leq 2^{r} E_{n, p}(f)+\frac{1}{(n+1)^{r}}\left(\frac{n}{2}\right)^{r} \omega_{r}\left(T, \frac{\pi}{n}\right)_{p} \\
& =\left(2^{r}+1\right) E_{n, p}(f)+\frac{1}{2^{r}} \omega_{r}\left(f, \frac{\pi}{n}\right)_{p} \leq\left(2^{r}+1\right) E_{n, p}(f)+\omega_{r}\left(f, \frac{\pi}{n+1}\right)_{p} .
\end{aligned}
$$

From [9, Theorem 6.1], we know that

$$
E_{n, p}(f) \leq \sqrt{r}(2 \ln (r)+12) \frac{([r / 2] !)^{2}}{r !} \omega_{r}\left(f, \frac{\pi}{n+1}\right)_{p} .
$$

It is known that (see [13]), for each $k \in \mathbb{N}$,

$$
\frac{(k !)^{2}}{(2 k) !}<\frac{\sqrt{\pi(k+1 / 2)}}{2^{2 k}}=\frac{\sqrt{\pi(2 k+1)}}{2^{2 k} \sqrt{2}} .
$$

Thus, if $r=2 k$, then

$$
E_{n, p}(f) \leq 2 \sqrt{r}(\ln (r)+6) \frac{\sqrt{r \pi}}{2^{r}} \omega_{r}\left(f, \frac{\pi}{n+1}\right)_{p} \leq \frac{8 r}{2^{r+1}}(6+\ln r) \omega_{r}\left(f, \frac{\pi}{n+1}\right)_{p}
$$

and

$$
\left\|\left(I-\sigma_{n}\right)^{r}(f)\right\|_{p} \leq(1+8 r(6+\ln r)) \omega_{r}\left(f, \frac{\pi}{n+1}\right)_{p}
$$


Remark 2.1. In [8], Ditzian and Ivanov said that the investigation of the rate of convergence of $\left(I-\sigma_{n}\right)^{r}(f)$ to 0 can be handled via some Riesz means, but no details were given. Anyway, the estimate in [8] for the typical means were presented in terms of a $K$-functional. Our approach is direct, and do not use $K$-functionals.

\section{SimultaneOUS APPROXIMATION}

Let us recall a Favard theorem.

Theorem 3.3. ([15, p. 289, ]) If $1 \leq p \leq \infty, r, n \in \mathbb{N}$ and $g \in W_{p}^{r}$, then

$$
E_{n, p}(g) \leq \frac{\pi}{2(n+1)^{r}} E_{n, p}\left(D^{r}(g)\right)
$$

and

$$
E_{n, p}(\widetilde{g}) \leq \frac{\pi}{2(n+1)^{r}} E_{n, p}\left(D^{r}(g)\right) .
$$

Theorem 3.3 is not written in the usual form of the Favard theorem. But, as Czipszer and Freud noticed [6, page 37], the inequalities presented above can be deduced from the original Favard ones.

It seems that the main results in simultaneous approximation by trigonometric polynomials are due to Czipszer and Freud [6]. They presented the arguments for continuous function, but (as some authors usually do) they explained that all the results hold in $\mathbb{L}^{p}$ spaces [6, pages 49-51]. Previously, Freud [10] announced the estimate

$$
\left\|D(f)-D\left(T_{n}\right)\right\|_{\infty} \leq C_{r}\left(n^{r}\left\|f-T_{n}\right\|_{\infty}+E_{n, \infty}\left(D^{r}(f)\right)\right), \quad f \in C_{2 \pi}^{r},
$$

where $T_{n} \in \mathbb{T}_{n}$ is the polynomial of the best approximation for $f$.

Here, we prefer to include a complete proof of Theorem 3.4 for several reasons. First, in [6] several details are omitted for $X^{p}$ with $1 \leq p<\infty$ and no information is included concerning the constants. It follows from our proof that the constants in Theorem 3.4 are not the best possible, but in application to the analysis of Fejér operators they provide reasonable estimates.

For the proofs, we need some auxiliary operators. The original idea goes back to de la Vallée Poussin [7].

Definition 3.1. Fix $r \in \mathbb{N}$. For $n \in \mathbb{N}, n \geq r$, and $f \in X^{1}$ define

$$
C_{n, r}(f, x)=\frac{1}{[n(1+1 / r)]-n} \sum_{k=n}^{[n(1+1 / r)]-1} S_{k}(f, x) .
$$

Taking into account that $\widetilde{S}_{n}(f, x)=\sum_{k=1}^{n} B_{k}(f, x)$, we also set

$$
\widetilde{C}_{n, r}(f, x)=\frac{1}{[n(1+1 / r)]-n} \sum_{k=n}^{[n(1+1 / r)]-1} \widetilde{S}_{k}(f, x) .
$$

We collect some properties of the sums $C_{n, r}$. The results are taken from [6, page 46].

Proposition 3.5. Fix $r \in \mathbb{N}$ and set $q=1+1 / r$. If $f \in \mathbb{L}^{1}, n \in \mathbb{N}$, and

$$
I_{n, r}(x)=\frac{1}{[n / r]} \frac{\sin ([n(2+1 / r)] t / 2) \sin ([n / r] t / 2)}{\sin ^{2}(t / 2)},
$$

then $C_{n, r}(f) \in \mathbb{T}_{[n q]-1}$ and

$$
C_{n, r}(f, x)=\frac{1}{2 \pi} \int_{-\pi}^{\pi} f(x+t) I_{n, r}(t) d t .
$$


Proposition 3.6. If $r, n \in \mathbb{N}, n \geq r, f, \widetilde{f} \in \mathbb{L}^{1}, g \in W_{1}^{1}$ and $T_{n} \in \mathbb{T}_{n}$, then

$$
C_{n, r}(\tilde{f}, x)=\widetilde{C}_{n, r}(f, x), \quad C_{n, r}\left(g^{\prime}, x\right)=\frac{d}{d x} C_{n, r}(g, x)
$$

and $C_{n, r}\left(T_{n}, x\right)=T_{n}(x)$.

The next result gives an estimate of the norm of the operator $C_{n, r}$.

Proposition 3.7. If $r, n \in \mathbb{N}$, and $n \geq r$, then

$$
\frac{1}{2 \pi} \int_{-\pi}^{\pi}\left|I_{n, r}(t)\right| d t \leq 3+\frac{1}{r}+\ln (2 r) .
$$

Proof. First, we write

$$
\frac{1}{2 \pi} \int_{-\pi}^{\pi}\left|I_{n, r}(t)\right| d t=\frac{2}{\pi} \int_{0}^{\pi / 2}\left|I_{n, r}(2 t)\right| d t .
$$

We split the interval $[0, \pi / 2]$ by considering $A_{1}=[0, \pi /(2 n)]$,

$$
A_{2}=[\pi /(2 n), \pi /(2[n / r])] \quad \text { and } \quad A_{3}=[\pi /(2[n / r]), \pi / 2] .
$$

Notice that

$$
1 \leq n / r \leq n \quad \text { and } \quad 1 \leq[n / r] \leq n .
$$

Taking into account that $|\sin (n x)| \leq n \sin x$, we obtain

$$
\frac{2}{\pi} \int_{A_{1}}\left|I_{n, r}(2 t)\right| d t \leq \frac{2}{\pi[n / r]}[n(2+1 / r)][n / r] \int_{0}^{\pi /(2 n)} d t \leq 2+1 / r .
$$

On the other hand, since $2 x \leq \pi \sin x$, one has

$$
\begin{gathered}
\frac{2}{\pi} \int_{A_{2}}\left|I_{n, r}(2 t)\right| d t \leq \frac{2}{\pi[n / r]} \frac{\pi}{2}[n / r] \int_{\pi /(2 n)}^{\pi /(2[n / r])} \frac{d t}{t} \\
=\ln \frac{n}{[n / r]}<\ln 2+\ln r
\end{gathered}
$$

because if $n \geq \max \{2, r\}$ and $n=q r+\theta$, with $q \in \mathbb{N}$ and $\theta \in[0,1)$, then $2 \theta<2 \leq n=q r+\theta$. Hence, $q r>\theta$ and $n=q r+\theta<2 r q=2 r[n / r]$.

Finally,

$$
\begin{gathered}
\frac{2}{\pi} \int_{A_{3}}\left|I_{n, r}(2 t)\right| d t \leq \frac{2}{\pi[n / r]}\left(\frac{\pi}{2}\right)^{2} \int_{\pi /(2[n / r])}^{\pi / 2} \frac{d t}{t^{2}} \\
\leq \frac{2}{\pi[n / r]}\left(\frac{\pi}{2}\right)^{2} \frac{2[n / r]}{\pi}=1 .
\end{gathered}
$$

Therefore,

$$
\frac{1}{2 \pi} \int_{-\pi}^{\pi}\left|I_{n, r}(t)\right| d t \leq 3+\frac{1}{r}+\ln (2 r) .
$$

Proposition 3.8. If $f \in X^{p}(1 \leq p \leq \infty)$, and $n, r \in \mathbb{N}, n \geq \max \{2, r\}$, then

$$
\left\|f-C_{n, r}(f)\right\|_{p} \leq\left(4+\frac{1}{r}+\ln (2 r)\right) E_{n, p}(f) .
$$

Proof. If $T \in \mathbb{T}_{n}$ and $\|f-T\|_{p}=E_{n, p}(f)$, then

$$
\begin{aligned}
& \left\|f-C_{n, r}(f)\right\|_{p}=\left\|f-T-C_{n, r}(f-T)\right\|_{p} \\
& \quad \leq\left(1+\left\|C_{n, r}\right\|_{1}\right)\|f-T\|_{p} \leq\left(4+\frac{1}{r}+\ln (2 r)\right) E_{n, p}(f) .
\end{aligned}
$$


Theorem 3.4. Assume $1 \leq p \leq \infty, r, n \in \mathbb{N}$ and $n \geq \max \{2, r\}$.

(i) If $g \in W_{p}^{r}, T \in \mathbb{T}_{n}$, and $\|g-T\|_{p}=E_{n, p}(g)$, then

$$
\left\|D^{r}(g)-D^{r}(T)\right\|_{p} \leq\left(4+\frac{1}{r}+\ln (2 r)\right)\left(1+\frac{e \pi}{2}\right) E_{n, p}\left(D^{r}(g)\right) .
$$

(ii) If $g, \widetilde{g} \in W_{p}^{r}, T \in \mathbb{T}_{n}$, and $\|g-T\|_{p}=E_{n, p}(g)$, then

$$
\left\|D^{r}(\widetilde{g})-D^{r}(\widetilde{T})\right\|_{p} \leq\left(4+\frac{1}{r}+\ln (2 r)\right)\left(E_{n, p}\left(D^{r}(\widetilde{g})\right)+\frac{e \pi}{2} E_{n, p}\left(D^{r}(g)\right)\right) .
$$

Proof. (i) Let $C_{n, r}$ be given as in Definition 3.1. Notice that

$$
([n(1+1 / r)]-1)^{r}<n^{r}\left(1+\frac{1}{r}\right)^{r}<e n^{r}, \quad n \geq r .
$$

From Propositions 3.7, 3.8 and 3.6, we obtain the following inequalities

$$
\begin{gathered}
\left\|C_{n, r}(g)-T\right\|_{p}=\left\|C_{n, r}(g-T)\right\|_{p} \leq\left(4+\frac{1}{r}+\ln (2 r)\right)\|g-T\|_{p} \\
\leq\left(4+\frac{1}{r}+\ln (2 r)\right) \frac{\pi}{2(n+1)^{r}} E_{n, p}\left(g^{(r)}\right), \\
\left\|D^{r}(g)-C_{n, r}\left(D^{r}(g)\right)\right\|_{p} \leq\left(4+\frac{1}{r}+\ln (2 r)\right) E_{n, p}\left(D^{r}(g)\right),
\end{gathered}
$$

and

$$
\begin{gathered}
\left\|D^{r}\left(C_{n, r}(g)\right)-D^{r}(T)\right\|_{p} \leq([n(1+1 / r)]-1)^{r}\left\|C_{n, r}(g)-T\right\|_{p} \\
\leq \frac{e \pi}{2}\left(4+\frac{1}{r}+\ln (2 r)\right) E_{n, p}\left(D^{r}(g)\right) .
\end{gathered}
$$

Therefore,

$$
\begin{gathered}
\left\|D^{r}(g)-D^{r}(T)\right\|_{p} \leq\left\|D^{r}(g)-C_{n, r}\left(D^{r}(g)\right)\right\|_{p}+\left\|D^{r}\left(C_{n, r}(g)\right)-D^{r}(T)\right\|_{p} \\
\leq\left(4+\frac{1}{r}+\ln (2 r)\right)\left(1+\frac{e \pi}{2}\right) E_{n, p}\left(D^{r}(g)\right) .
\end{gathered}
$$

(ii) For the conjugate function, we consider the relations

$$
C_{n, r}\left(D^{r}(\widetilde{g})\right)=D^{r}\left(\widetilde{C}_{n, r}(g)\right)
$$

and

$$
\begin{aligned}
\| D^{r}(\widetilde{g}) & -D^{r}(\widetilde{T})\left\|_{p} \leq\right\| D^{r}(\widetilde{g})-C_{n, r}\left(D^{r}(\widetilde{g})\right)\left\|_{p}+\right\| D^{r}\left(\widetilde{C}_{n, r}(g)-\widetilde{T}\right) \|_{p} \\
& \leq\left(4+\frac{1}{r}+\ln (2 r)\right) E_{n, p}\left(D^{r}(\widetilde{g})\right)+e n^{r}\left\|C_{n, r}(g)-T\right\|_{p} \\
& \leq\left(4+\frac{1}{r}+\ln (2 r)\right)\left(E_{n, p}\left(D^{r}(\widetilde{g})\right)+\frac{e \pi}{2} E_{n, p}\left(D^{r}(g)\right)\right),
\end{aligned}
$$

where we have used Theorem 2.1. 


\section{VORONOVSKAYA-TYPE THEOREMS}

Zamansky [16, Th. 14] proved that if $f \in C_{2 \pi}^{1}$, then

$$
\lim _{n \rightarrow \infty}\left\|(n+1)\left(\widetilde{\sigma}_{n}(f)-\widetilde{f}\right)-D(f)\right\|_{\infty}=0 .
$$

Later he verified that [17, Theorem 1], if $E_{n}(f)_{\infty} \leq C / n^{r}, r>1$, then

$$
\lim _{n \rightarrow \infty}\left\|n\left(\sigma_{n}(f)-f\right)+D(\widetilde{f})\right\|_{\infty}=0 .
$$

The extension of the last inequality to $\mathbb{L}^{p}$ spaces, $1<p<\infty$, was given by Butzer and Görlich in [4, page 386]. The result is presented in Corollary 4.1 below. We remark that the proof presented below is simpler than the ones of Zamansky and it provides the rate of convergence. We consider first the Fejér operators and later the iterative combination.

As usual, we set

$$
S_{n}(f, x)=\sum_{k=0}^{n} A_{k}(f, x)
$$

for the partial sums of the Fourier series of $f$.

Theorem 4.5. If $1 \leq p \leq \infty, f, \tilde{f} \in W_{p}^{1}$, and $n>1$, then

$$
\left\|(n+1)\left(\sigma_{n}(f)-f\right)+D(\widetilde{f})\right\|_{p} \leq(1+3 e) \pi E_{n, p}(D(f))+6 E_{n, p}(D(\widetilde{f}))
$$

and

$$
\left\|(n+1)\left(\widetilde{\sigma}_{n}(f)-\widetilde{f}\right)-D(f)\right\| \leq(1+3 e) \pi E_{n, p}(D(\widetilde{f}))+6 E_{n, p}(D(f)) .
$$

Proof. If $T_{n} \in \mathbb{T}_{n}$ satisfies $E_{n, p}(f)=\left\|f-T_{n}\right\|_{p}$, then taking into account (2.7), Theorem 3.4 and equation (3.8) one has

$$
\begin{array}{r}
\left\|\sigma_{n}(f)-f+\frac{D(\widetilde{f})}{n+1}\right\|_{p}=\left\|\sigma_{n}\left(f-T_{n}\right)-\left(f-T_{n}\right)+\frac{D(\tilde{f})-D\left(\widetilde{T}_{n}\right)}{n+1}\right\|_{p} \\
\leq 2 E_{n, p}(f)+\frac{5+\ln (2)}{n+1}\left(E_{n, p}(D(\widetilde{f}))+\frac{e \pi}{2} E_{n, p}(D(f))\right) \\
\leq \frac{\pi}{n+1} E_{n, p}(D(f))+\frac{5+\ln (2)}{n+1}\left(E_{n, p}(D(\widetilde{f}))+\frac{e \pi}{2} E_{n, p}(D(f))\right) \\
\leq(1+3 e) \frac{\pi}{(n+1)} E_{n, p}(D(f))+\frac{6}{n+1} E_{n, p}(D(\widetilde{f})) .
\end{array}
$$

Set $g=\widetilde{f}$. Let us verify that $\widetilde{\sigma}_{n}(f)=\sigma_{n}(g)$ a.e. and $D(\widetilde{g})(x)=-D(f)(x)$ a.e. In fact, if

$$
f(x) \sim \frac{a_{0}(f)}{2}+\sum_{k=1}^{\infty}\left(a_{k} \cos (k x)+b_{k}(f) \sin (k x)\right),
$$

then

$$
\widetilde{f}(x) \sim \sum_{k=1}^{\infty}\left(-b_{k} \cos (k x)+a_{k}(f) \sin (k x)\right) .
$$

Thus,

$$
\widetilde{\sigma}_{n}(f, x)=\sum_{k=1}^{n}\left(1-\frac{k}{n+1}\right)\left(-b_{k} \cos (k x)+a_{k}(f) \sin (k x)\right)=\sigma_{n}(\widetilde{f}, x) .
$$


On the other hand,

$$
\begin{gathered}
D(f)(x) \sim \sum_{k=1}^{\infty} k\left(b_{k} \cos (k x)-a_{k}(f) \sin (k x)\right), \\
\widetilde{g}(x) \sim-\sum_{k=1}^{\infty}\left(a_{k} \cos (k x)+b_{k}(f) \sin (k x)\right)
\end{gathered}
$$

and

$$
D(\widetilde{g})(x) \sim-\sum_{k=1}^{\infty} k\left(-b_{k} \cos (k x)+a_{k}(f) \sin (k x)\right) \sim-D(f)(x) .
$$

Therefore

$$
\widetilde{\sigma}_{n}(f)-\widetilde{f}-\frac{D(f)}{n+1}=\sigma_{n}(g)-g+\frac{D(\widetilde{g})}{n+1} .
$$

Since $g=\widetilde{f} \in W_{p}^{1}$ and $\widetilde{g}=-f \in W_{p}^{1}$, one has

$$
\begin{gathered}
\left\|(n+1)\left(\widetilde{\sigma}_{n}(f)-\widetilde{f}\right)+D(f)\right\| \leq \pi(1+3 e) E_{n, p}(D(g))+6 E_{n, p}(D(\widetilde{g})) \\
=\pi(1+3 e) E_{n, p}(D(\widetilde{f}))+6 E_{n, p}(D(f))
\end{gathered}
$$

and this proves the result.

Corollary 4.1 is a simple consequence of the previous result. Recall that, for $1<p<\infty$, the element $\widetilde{f}$ always exists.

Corollary 4.1. If $1<p<\infty$ and $f \in W_{p}^{1}$, then

$$
\lim _{n \rightarrow \infty}\left\|(n+1)\left(\sigma_{n}(f)-f\right)-D(\tilde{f})\right\|_{p}=0 .
$$

A result similar to Theorem 4.5 can be proved for the linear combination $\left(I-\sigma_{n}\right)^{r}$ of Fejér operators. Here, we only consider the case of even $r$.

Theorem 4.6. If $1 \leq p \leq \infty, r \in \mathbb{N}$ is even, $f \in W_{p}^{r}$, and $n \geq r$, then

$$
\left\|(n+1)^{r}\left(I-\sigma_{n}\right)^{r}(f)-(-1)^{r / 2} D^{r}(f)\right\|_{p} \leq\left(2^{r+1}+7(5+\ln (2 r))\right) E_{n, p}\left(D^{r}(f)\right) .
$$

Proof. If $T \in \mathbb{T}_{n}$ is chosen from the condition $E_{n, p}(f)=\|f-T\|_{p}$, from Proposition 2.4 one has

$$
\left(I-\sigma_{n}\right)^{r}(f)-\frac{(-1)^{r / 2}}{(n+1)^{r}} D^{r}(f)=\left(I-\sigma_{n}\right)^{r}(f-T)-\frac{(-1)^{r / 2}}{(n+1)^{r}} D^{r}(f-T),
$$

and it follows from (3.8) and Theorem 3.4 (here the condition $n \geq r$ is needed) that

$$
\begin{aligned}
& \left\|\left(I-\sigma_{n}\right)^{r}(f)-\frac{(-1)^{r / 2}}{(n+1)^{2}} D^{r} f\right\|_{p} \leq 2^{r} E_{n, p}(f)+\frac{1}{(n+1)^{r}}\left\|D^{r}(f-T)\right\|_{p} \\
& \quad \leq \frac{2^{r-1} \pi}{(n+1)^{r}} E_{n, p}\left(D^{r}(f)\right)+\left(4+\frac{1}{r}+\ln (2 r)\right)\left(1+\frac{e \pi}{2}\right) \frac{1}{(n+1)^{r}} E_{n, p}\left(D^{r}(f)\right) \\
& \quad \leq \frac{2^{r-1} \pi}{(n+1)^{r}} E_{n, p}\left(D^{r}(f)\right)+(5+\ln (2 r)) \frac{(1+2 e)}{(n+1)^{r}} E_{n, p}\left(D^{r}(f)\right) .
\end{aligned}
$$




\section{REFERENCES}

[1] T. Acar, A. Aral, and I. Raşa: The new forms of Voronovskaya's theorem in weighted spaces. Positivity 20 (2016), 25-40.

[2] A. Aral, H. Gonska, M. Heilmann, and G. Tachev: Quantitative Voronovskaya-type results for polynomially bounded functions. Results. Math. 70 (2016), 313-324.

[3] J. Bustamante: Direct and strong converse inequalities for approximation with Fejér means. to appear.

[4] P. L. Butzer and E. Gorlich: Saturationsklassen und asymptotische Eigenschten trigonometrischer singulärer Integrale. Festschrift zur Gedächtnisfeier für Karl Weierstraß 1815-1965Arbeitsgemeinschaft für Forschung des Landes Nordrhein-Westfalen, Bd. 33, Köln (1966), 339-392.

[5] P. L. Butzer, R. J. Nessel: Fourier Analysis and Approximation. New York-Base1 (1971).

[6] J. Czipszer, G. Freud: Sur l'approximation d'une fonction périodique et de ses dérivées successives par un polynome trigonometrique et par ses dérivées succesives. Acta Math. 99 (1958), 33-51.

[7] C. de la Vallée Poussin: Leçons sur l'Approximation des fonctions d'une variable réelle. Paris, Gauthier-Villars, 1919.

[8] Z. Ditzian, K. Ivanov: Strong converse inequalities. J. Analyse Math. 61 (1993), 61-111.

[9] S. Foucart, Y. Kryakin and A. Shadrin, On the exact constant in the Jackson-Stechkin inequality for the uniform metric: Constr. Approx. 29 (2009), 157-179.

[10] G. Freud: Über gleichzeitige Approximation einer Funktion und ihrer Derivierten. Intern. Math. Nachrichten, Wien, 47/49 (1957), 36-37.

[11] H. Gonska: On the degree of approximation in Voronovskaja's theorem. Studia Univ. Babeş-Bolyai, Mathematica 52 (3) (2007), 103-116.

[12] H. Gonska, I. Raşa: Remarks on Voronovskaya's theorem, General Mathematics 16 (4) (2008), 87-97.

[13] D. K. Kazarinoff: On Wallis formula. Edinburgh Math. Notes 40 (1956), 19-21.

[14] N. P. Korneichuk, V. F. Babenko and A. A. Ligun: Extremal Properties of Polynomials and Splines. (Russian) Naukova Dumka, Kiev, 1992.

[15] A. F. Timan: Theory of Approximation of Functions of Real Variable. Pergamon Press, 1963.

[16] M. Zamansky: Classes de saturation de certains procédés d'approximation des séries de Fourier des fonctions continues et application à quelques problèmes d'approximation. Ann. Sci. Ecole Normale Sup. 3 (66) (1949), 19-93.

[17] M. Zamansky: Classes de saturation des procédés de sommation des séries de Fourier et application aux séries trigonométriques. Ann. Sci. Ecole Normale Sup. 67 (1950), 161-198.

Benemérita Universidad Autónoma de Puebla

DEPARTMENT OF MATHEMATICS

PUEBLA, MÉXICO

ORCID: 0000-0003-2856-6738

E-mail address: jbusta@fcfm.buap.mx 